\title{
A Method for Measuring the Specific Electrical Conductivity of an Anisotropically Conductive Medium
}

\author{
S. Ašmontas ${ }^{a, *}$, J. KLEIZA $^{b}$ And V. KleizA ${ }^{a}$ \\ ${ }^{a}$ Kaunas Technology University, Panevėžys Institute \\ Klaipėdos 1, 35209 Panevėžys, Lithuania \\ ${ }^{b}$ Vilnius Gediminas Technical University \\ Saulètekio al. 11, 10223 Vilnius, Lithuania
}

(Received May 31, 2007; in final form February 8, 2008)

\begin{abstract}
The paper deals with the ways of finding an electrical conductivity tensor of a plane and anisotropically conductive sample. Application of the Van der Pauw method to investigate the conductivity of anisotropically conductive media makes the basis of research. Several models of distribution of the electric field potential are presented, their merits and demerits are discussed, and the necessary physical measurements are indicated. On the basis of these models, the respective calculation expressions of the specific conductivity tensor are derived and algorithms for their realization and error calculation are developed.
\end{abstract}

PACS numbers: 06.20.Dk, 02.60.Cb

\section{Introduction}

The methods for determining electrical conductivity of thin (metal) films are topical in practice, whereas many materials of interest are of a crystalline structure and therefore are likely to be anisotropic [1-4]. Van der Pauw (1958) [5] made an important contribution to the development of these methods. With the use of his method we can calculate electrical conductivity of an isotropically conductive sample with 4 point contacts of uniform thickness and any geometrical form. To realize this method, it suffices to measure the difference of two potentials and current intensity. Then, having solved a transcendental equation, not very complicated in the mathematical sense, we can find the specific conductivity of the substance of sample. Later on, in 1972, the paper of Price [6] appeared in which it is proved that, under the anisotropic conductivity, the solution to the Van

*corresponding author; e-mail: asmontas@pfi.lt 
der Pauw equation is coincident with the root of the determinant of conductivity tensor.

This work encompasses a more extensive problem: to establish all the summands of the specific conductivity tensor of an anisotropically conductive slab. It has been proved in the work that, if it is a rectangular-shaped sample with 4 point contacts infixed at its angles and with the fifth one in one of the sides, then, after making 3 measurements, analogous to the Van der Pauw method, we can build a system of equations that is solved uniquely, and its solution is the summands of the conductivity tensor. In addition, a numerical algorithm has been constructed for solving this problem, and errors of the method have been analyzed, the contacts of sample being of finite length.

\section{The method for calculating the summands} of the conductivity tensor

In this section, we define physical measurements, that are sufficient for establishing the specific conductivity tensor $\sigma$, and indicate its calculating method.

The experiments are done using a rectangular shaped sample $(a \times b)$ of equal height $d$ with five point contacts: 4 of them (1-4) are located at angular points, and the fifth one in one of its sides (Fig. 1).

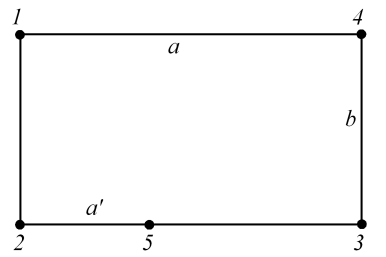

Fig. 1. Contact arrangement in sample and the distances $a, a^{\prime}, b$ among them.

Three experiments were performed:

1. After inducing voltage between contacts 1 and 2 , we measure the flowing current intensity $I_{12}$ and appearing differences of potentials $\Delta \varphi_{34}=\varphi_{4}-\varphi_{3}$ (Fig. 2a);

2. After inducing voltage between contacts 1 and 2 , we measure the flowing current intensity $I_{12}$ and appearing differences of potentials $\Delta \varphi_{53}=\varphi_{3}-\varphi_{5}$ (Fig. 2b);

3. After inducing voltage between contacts 1 and 4 , we measure the flowing current intensity $I_{14}$ and the appearing difference of potentials $\Delta \varphi_{32}=\varphi_{2}-\varphi_{3}$ (Fig. 2c).

We show how to calculate the summands of the specific conductivity tensor $\sigma$ in quest (see Sect. 3.1), based on the results of experiments done.

At the first stage, we solve the Van der Pauw equation [5]:

$\exp \left(-\pi s d \frac{\Delta \varphi_{34}}{I_{12}}\right)+\exp \left(-\pi s d \frac{\Delta \varphi_{32}}{I_{14}}\right)=1$, 

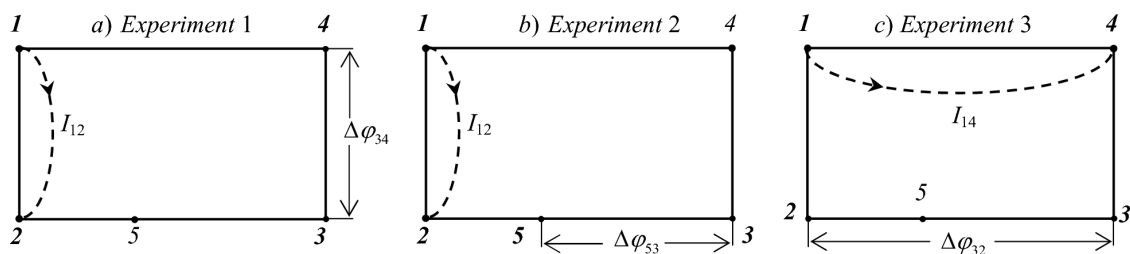

Fig. 2. Measurements carried out in the experiments.

and find an unknown $s$ which is coincident [6], in the physical sense, with the root of the determinant $s=\sqrt{\operatorname{det} \sigma}$.

This stage is analogous to the Van der Pauw method, however, in this case, we calculate not the specific conductivity of anisotropic substance, but a determinant of the specific conductivity tensor.

At the next stage, applying the formulae

$$
\begin{aligned}
& k=\exp \left(-\pi d s \frac{\Delta \varphi_{34}}{I_{12}}\right), \\
& t=\exp \left(-\pi d s \frac{\Delta \varphi_{53}}{I_{12}}\right),
\end{aligned}
$$

we establish auxiliary parameters $0<k<1$ and $0<t<1$. Having calculated them, we form and solve an equation of one unknown $\alpha$ :

$$
\frac{\int_{0}^{t} f_{\alpha, k} \mathrm{~d} \tau}{A_{\alpha, k}}=\frac{a^{\prime}}{a},
$$

here $f_{\alpha, k}=\tau^{\alpha-1}(1-\tau)^{-\alpha}(1-k \tau)^{\alpha-1}, A_{\alpha, k}=\int_{0}^{1} f_{\alpha, k} \mathrm{~d} \tau$, and $a, a^{\prime}$ are distances between contacts $1-4$ and $2-5$.

The solution $\alpha(0<\alpha<1)$ of Eq. (4) and the parameters $k, t$ and $\sqrt{\operatorname{det} \sigma}$ are sufficient to determine the tensor summands $\sigma_{11}, \sigma_{12}, \sigma_{22}$.

At the third, final, stage, the $\sigma_{11}, \sigma_{12}, \sigma_{22}$ are calculated by the formulae

$$
\sigma_{11}=\lambda \frac{a}{b} \frac{A_{\alpha, 1-k}}{A_{\alpha, k}}, \quad \sigma_{12}=-\lambda \cos (\alpha \pi), \quad \sigma_{22}=\lambda \frac{b}{a} \frac{A_{\alpha, k}}{A_{\alpha, 1-k}},
$$

where $\lambda=\sqrt{\operatorname{det} \sigma} / \sin (\alpha \pi)$.

In the following section, we will ground this method of tensor calculation, and here we restrict ourselves only to the remarks on numerical realization of the method. While considering the latter, we face the following problems of computational mathematics:

1. solution of Eq. (1) to calculate the tensor determinant;

2. formation and application of numerical procedures, to compute integrals $\int_{0}^{t} f_{\alpha, k} \mathrm{~d} \tau$

3. solution of Eq. (4) to calculate the parameters $\alpha$.

The authors in [7] have constructed special numerical algorithms for solving all these problems, in the application of which only elementary functions are 
used. However, in order that every researcher could employ the method under consideration, we describe it by applying the mathematical package Maple. This is widespread package that enables us to solve various mathematical problems. Its internal operators (int) allow for prompt highly accurate computations of the integrals $\int_{0}^{t} f_{\alpha, k} \mathrm{~d} \tau$ (beginning with its 7 th version of Maple, several hundreds of integrals per second with an accuracy of $10^{-14}$ ). These integrals are indispensable in solving Eq. (4). Moreover, it has been proved [8] that its left-hand side is a monotone function of the unknown $\alpha$. Therefore, it is convenient to solve it by the bisection method because it converges unconditionally, and its error is $\varepsilon \approx 10^{-m / 3}$ ( $m$ is the number of iterations). Therefore, having computed e.g. 60 integrals, we define the value of the parameter $\alpha$ with an accuracy of $10^{-10}$. We can find the other unknown det $\sigma$, defined by the Van de Pauw Eq. (1), directly using the operator fsolve.

Let us note that the time of all the operations (1)-(5) necessary to realize the method makes up about 1 second.

\section{Foundation of the method}

\subsection{Reduction to an isotropic case}

Distribution of the electric field potential $\varphi(x, y)$ in a plain domain $G$, the electrical conductivity of which is defined by the tensor

$$
\sigma=\left(\begin{array}{ll}
\sigma_{11} & \sigma_{12} \\
\sigma_{12} & \sigma_{22}
\end{array}\right)=\text { const, } \quad \sigma_{11}, \sigma_{22}, \operatorname{det} \sigma=\sigma_{11} \sigma_{22}-\left(\sigma_{12}\right)^{2}>0
$$

is expressed by a boundary problem

$$
\left\{\begin{array}{l}
\operatorname{div} \boldsymbol{j}=0, \quad(x, y) \in G, \\
\boldsymbol{j}=\sigma \operatorname{grad} \varphi \\
\left.\varphi\right|_{\kappa}=\mathrm{const}, \\
\left.(\sigma \operatorname{grad} \varphi)_{n}\right|_{\Gamma \backslash \kappa}=0,
\end{array}\right.
$$

here $\Gamma$ is the contour of domain $G, \kappa$ is the part of the contour coincident with the contacts $\kappa_{1}, \kappa_{2}, \ldots$, and $n$ is the contour normal (Fig. 3a).
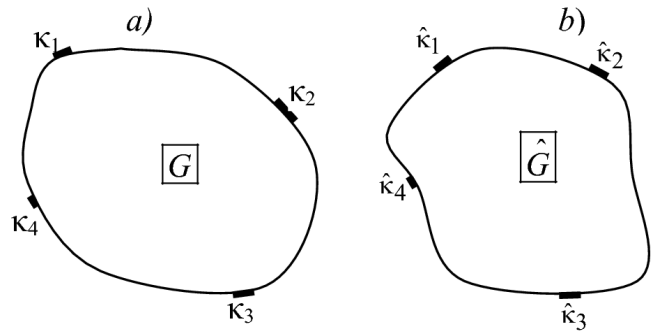

Fig. 3. Domain transformation scheme: (a) initial domain; (b) transformed domain. 
After replacing variables in problem (7):

$$
\xi=x \sigma_{22}-y \sigma_{12}, \quad \eta=y \sqrt{\operatorname{det} \sigma},
$$

we get a boundary problem for the function $\hat{\varphi}(\xi, \eta)=\varphi(x, y)$ in the respective domain $\hat{G}$ (Fig. 3b) of the Laplace equation

$$
\left\{\begin{array}{l}
\operatorname{div} \hat{j} \equiv \Delta \hat{\varphi}(\xi, \eta)=0, \quad(\xi, \eta) \in \hat{G}, \\
\hat{j}=\operatorname{sgrad} \hat{\varphi}, \\
\left.\hat{\varphi}\right|_{\hat{\kappa}}=\text { const, } \\
\left.\frac{\partial \hat{\varphi}}{\partial \hat{n}_{\xi, \eta}}\right|_{\hat{\Gamma} \backslash \hat{\kappa}}=0,
\end{array}\right.
$$

where $\hat{n}_{\xi \eta}$ is the contour normal of domain $\hat{G}$.

In the physical sense, Eq. (9) defines the potential distribution on isotropic sample $\hat{G}$, therefore it suffices to determine its conductivity $s$. To this end, we prove the statement: if the numbers $s=\sqrt{\operatorname{det} \sigma}$ in problem (9), then problems (7) and (9) are equivalent.

\subsection{Mapping to a half-plane}

Let the shape of an anisotropically conductive sample be a rectangle $G=$ $\{a \times b\}$ (Fig. 1), the sides of which are arbitrarily oriented with respect to tensor principal directions. After transforming (8), we get that the mapping $(\xi, \eta)$ of domain $G$ on the plane is a parallelogram with the side lengths $L_{1}=a \sigma_{22}, L_{2}=$ $b \sqrt{\sigma_{11} \sigma_{22}}$, and the angle between them

$$
\alpha \pi=\arccos \frac{-\sigma_{12}}{\sqrt{\sigma_{11} \sigma_{22}}} .
$$

Besides, the integral

$$
\xi+\mathrm{i} \eta=\mu \int_{0}^{z} f_{\alpha, k}(\tau) \mathrm{d} \tau
$$

here $f_{\alpha, k}(\tau)=\tau^{\alpha-1}(1-\tau)^{-\alpha}(1-k \tau)^{\alpha-1}, \mu=a \sigma_{22}\left(\int_{0}^{1} f_{\alpha, k}(\tau) \mathrm{d} \tau\right)^{-1}$ conformally maps the half-plane $S$ : $z=t+\mathrm{i} \vartheta, \vartheta \geq 0$ into the parallelogram so that the mappings of points $t= \pm \infty, 0,1,1 / k$ are vertices $1,2,3,4$ of the parallelogram.

Now, basing on [5] and Fig. 4, we compute

$$
\begin{aligned}
& \frac{\Delta \varphi_{43}}{I_{12}}=\left.\frac{1}{\pi d s} \ln \left(\frac{(p+q)(q+r)}{q(p+q+r)}\right)\right|_{p=\infty, q=1, r=1 / k-1}=\frac{-1}{\pi d s} \ln k, \\
& \frac{\Delta \varphi_{32}}{I_{14}}=\left.\frac{1}{\pi d s} \ln \left(\frac{(p+q)(q+r)}{p r}\right)\right|_{p=\infty, q=1, r=1 / k-1}=\frac{-1}{\pi d s} \ln (1-k) .
\end{aligned}
$$

Hence $k=\exp \left(-\pi d s \frac{\Delta \varphi_{43}}{I_{12}}\right)$ and $1-k=\exp \left(-\pi d s \frac{\Delta \varphi_{32}}{I_{14}}\right)$. Thus, equality (2) is proved. By adding the latter equalities, we obtain the Van der Pauw Eq. (1). Reasoning analogously, we can get the mapping of contact 5 on the real axis $t$ (Fig. 2b): 


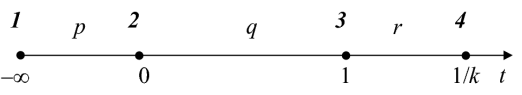

Fig. 4. Contact images and distances $p, q, r$ among them.

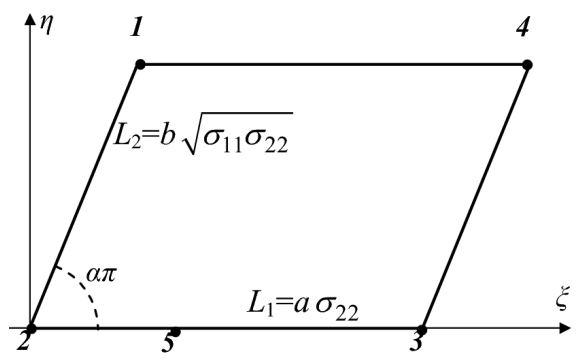

Fig. 5. Mapping of rectangular shape sample onto parallelogram.

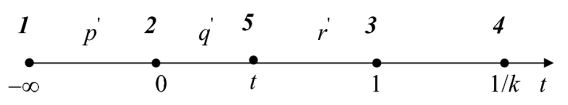

Fig. 6. Contact images and distances $p^{\prime}, q^{\prime}, r^{\prime}$ among them.

$$
\frac{\Delta \varphi_{53}}{I_{12}}=\left.\frac{1}{\pi d s} \ln \left(\frac{\left(p^{\prime}+q^{\prime}\right)\left(q^{\prime}+r^{\prime}\right)}{q^{\prime}\left(p^{\prime}+q^{\prime}+r^{\prime}\right)}\right)\right|_{p^{\prime}=\infty, q^{\prime}=t, r^{\prime}=1-t}=\frac{-1}{\pi d s} \ln t .
$$

Hence equality (3) is derived directly.

Since the position of contact 5 in the rectangle side is known (its distance from contact 2 is equal to $a^{\prime}$, Fig. 1), based on (8), (11), we have $\mu \int_{0}^{t} f_{\alpha, k}(\tau) \mathrm{d} \tau=a^{\prime} \sigma_{22}$ and $\mu \int_{0}^{1} f_{\alpha, k}(\tau) \mathrm{d} \tau=a \sigma_{22}$. This substantiates relation (4). It should be noted that the length of another parallelogram side $L_{2}$ (Fig. 5) can also be expressed by integral [7]:

$$
L_{2}=a \sqrt{\sigma_{11} \sigma_{22}}=\mu \int_{1}^{1 / k}\left|f_{\alpha, k}\right| \mathrm{d} \tau=\mu \int_{0}^{1} f_{\alpha, 1-k} \mathrm{~d} \tau=\mu A_{\alpha, 1-k} .
$$

From this and Eq. (10), the system

$$
\left\{\begin{array}{l}
\frac{-\sigma_{12}}{\sqrt{\sigma_{11} \sigma_{22}}}=\cos (\alpha \pi), \\
\sqrt{\frac{\sigma_{11}}{\sigma_{22}}}=\frac{a}{b} \frac{A_{\alpha, 1-k}}{A_{\alpha, k}} \\
\sigma_{11} \sigma_{22}-\sigma_{12}^{2}=\operatorname{det} \sigma
\end{array}\right.
$$

is constructed which has a unique solution (5) in the domain $\sigma_{11}, \sigma_{22}>0$.

\section{Simulation of experiments}

The formulae for computing tensor summands presented in the third section are exact only in case all the sample contacts are of zero length. Naturally, when 
doing experiments this condition will not be satisfied, and the tensor will be calculated only approximately. These errors will be estimated, if we build experiment imitating formulae.

Let us assume that the lengths of all the (1-4) angular contacts are $2 h$, while of the 5 th one it is $h$ (Fig. 7a). We shall find analytical expressions of the flowing current intensities and contact potentials if we perform the experiments described in the third section.

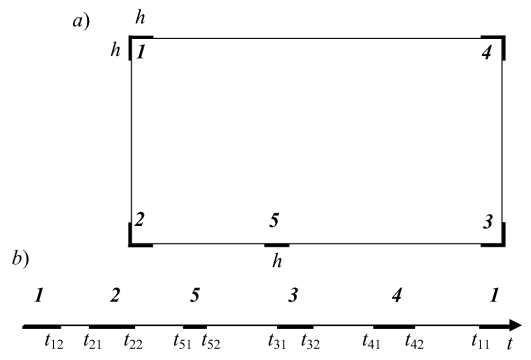

Fig. 7. Sample with finite contact length (a) and their images on real axis (b).

First let us consider the first experiment when the current $I_{12}$ is flowing through contacts 1-2. If the tensor is known, the mappings of points of the rectangular sample contour are also known: (8) onto the parallelogram $P$ and (11) - into the half-plane $S$. After mapping we obtain the maps of contacts $1-5$ on the real axis $t$ of the half-plane $S$. The coordinates of their boundary points are denoted as $t_{i j}$ (Fig. $7 \mathrm{~b}$ ).

Let us construct the function of argument $\tau$ :

$$
F=\frac{\left(T_{3}-\tau\right)\left(T_{4}-\tau\right)\left(T_{5}-\tau\right)}{\sqrt{-\prod_{i=1}^{5}\left(t_{i 1}-\tau\right)\left(t_{i 2}-\tau\right)}}
$$

and choose the numbers $T_{3}, T_{4}, T_{5}$ so that

$$
\int_{t_{i 1}}^{t_{i 2}} F \mathrm{~d} \tau=0, \quad T_{i} \in\left(t_{i 1}, t_{i 2}\right), \quad i=3,4,5 .
$$

Then the integral

$$
u+\mathrm{i} v=\int_{t_{12}}^{t} F \mathrm{~d} \tau
$$

conformally maps the half-plane $S$ onto a polygon $W$ (i.e., into a rectangle with 3 cuts, Fig. 8a). Since within the domain $W$ the potential $\varphi$ satisfies the Laplace equation all the contacts 1-5 arranged on the straight lines parallel to the imaginary axis $v$, the potential in them being constant, and at the remaining points of contour $W$ the derivative in the direction of the normal is equal to 0 , the potential in the domain $W$ varies linearly. In order to find the expression of potential $\varphi$, let us assume that voltage $V$ is induced between contacts 1 and 2: let the potential of contact 1 be $\varphi=V, 2-\varphi=0$. Then, in view of (13) we obtain 

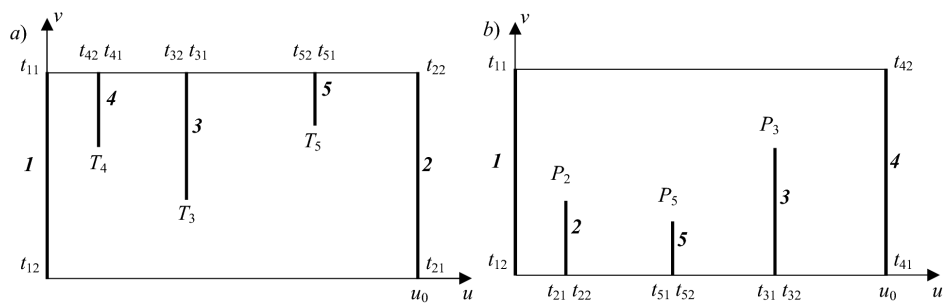

Fig. 8. The scheme of the polygon $W$. Points $t_{i j}$ and $T_{i}, P_{i}$ denote image points 1-5 after mapping (3.2) (a) and (3.4) (b).

$$
\varphi(u, v)=V\left(1-u / u_{0}\right)=V\left[1-\Re\left(\int_{t_{12}}^{t} F \mathrm{~d} \tau\right) / \int_{t_{12}}^{t_{21}} F \mathrm{~d} \tau\right] .
$$

We denote here by $\Re$ the real part of a complex number.

We compute the difference $\Delta \varphi_{34}=\varphi_{4}-\varphi_{3}$ of potentials induced between contacts 3 and 4 by integrating over the real axis segment $t_{32} t_{41}$ :

$$
\Delta \varphi_{34}=V \frac{\int_{t_{32}}^{t_{41}}|F| \mathrm{d} \tau}{\int_{t_{12}}^{t_{21}} F \mathrm{~d} \tau}
$$

and the flowing current intensity $I_{12}$ - over the real axis segment $t_{21} t_{22}$ :

$$
I_{12}=V d \sqrt{\operatorname{det} \sigma} \frac{\int_{t_{21}}^{t_{22}}|F| \mathrm{d} \tau}{\int_{t_{12}}^{t_{21}} F \mathrm{~d} \tau} .
$$

Hence we get the imitation formula

$$
\frac{\Delta \varphi_{34}}{I_{12}}=\frac{1}{d \sqrt{\operatorname{det} \sigma}} \frac{\int_{t_{32}}^{t_{41}}|F| \mathrm{d} \tau}{\int_{t_{21}}^{t_{22}}|F| \mathrm{d} \tau}
$$

of the first experiment (Fig. 2a).

By means of similar transformations, we obtain imitation formulae

$$
\begin{aligned}
\frac{\Delta \varphi_{53}}{I_{12}} & =\frac{1}{d \sqrt{\operatorname{det} \sigma}} \frac{\int_{t_{52}}^{t_{31}}|F| \mathrm{d} \tau}{\int_{t_{21}}^{t_{22}}|F| \mathrm{d} \tau}, \\
\frac{\Delta \varphi_{32}}{I_{14}} & =\frac{1}{d \sqrt{\operatorname{det} \sigma}} \frac{\int_{t_{22}}^{t_{51}}|\hat{F}| \mathrm{d} \tau+\int_{t_{52}}^{t_{31}}|\hat{F}| \mathrm{d} \tau}{\int_{t_{41}}^{t_{42}}|\hat{F}| \mathrm{d} \tau}
\end{aligned}
$$

of the second (Fig. 2b) and third (Fig. 2c) experiments. In the last formula

$$
\hat{F}=\frac{\left(P_{2}-\tau\right)\left(P_{3}-\tau\right)\left(P_{5}-\tau\right)}{\sqrt{-\prod_{i=1}^{5}\left(t_{i 1}-\tau\right)\left(t_{i 2}-\tau\right)}},
$$

numbers $P_{3}, P_{4}, P_{5}$ satisfy the equalities

$$
\int_{t_{i 1}}^{t_{i 2}} \hat{F} \mathrm{~d} \tau=0, \quad P_{i} \in\left(t_{i 1}, t_{i 2}\right), \quad i=2,3,5
$$

while the map of half-plane $S$, after mapping 


$$
u+\mathrm{i} v=\int_{t_{12}}^{t} \hat{F} \mathrm{~d} \tau
$$

is presented in Fig. 8b.

Let us note that, on the basis of integral expression (14) of the flowing current intensity, conditions (12): $\int_{t_{i 1}}^{t_{i 2}} F \mathrm{~d} \tau=0, i=3,4,5$ show that the general amount of the current flowing through the corresponding contacts $3,4,5$ (i.e., through those of them which are not coincident with the current contacts) is equal to 0 . Conditions (18) introduced above have the same sense.

\section{Error estimation}

Formulae (5) for calculating a tensor are derived in the second and third sections under the assumption that all the lengths of sample contacts are zero $(h=0)$, which is mathematical idealization of a real sample. Therefore, when computing the tensor $\sigma$ in this way, we shall find only its approximate value $\bar{\sigma}$. However, this is not the only source of errors of the method proposed: in the case of isotropic conductivity, the value of errors is influenced by geometrical parameters $a$ and $b$ of the sample, and in the anisotropic case, by the value of the tensor $\sigma$ summands in addition.

Thus, there arises an important problem for defining the error dependence on the parameters mentioned. With a view to solve it, experiment imitation formulae (15)-(17) have been derived in the fourth section. According to them, the exact value of tensor $\sigma$ being known, one can compute the quantities obtained during the experiment. Then, applying formulae (5) of the method, one can establish an approximate value of the tensor and, consequently, estimate the error.

We are doing that by defining a relative error

$$
\delta=\frac{\|\bar{\sigma}-\sigma\|}{\|\sigma\|} 100 \%
$$

where $\|\sigma\|=\sqrt{\sigma_{11}^{2}+\sigma_{12}^{2}+\sigma_{22}^{2}}$.

In order to briefly consider as many different cases as possible, let us note that for any tensor (6), the relation

$$
\sigma=\left(\begin{array}{cc}
\cos \theta & -\sin \theta \\
\sin \theta & \cos \theta
\end{array}\right)\left(\begin{array}{cc}
\sigma_{1} & 0 \\
0 & \sigma_{2}
\end{array}\right)\left(\begin{array}{cc}
\cos \theta & \sin \theta \\
-\sin \theta & \cos \theta
\end{array}\right), \quad \theta \in[0, \pi]
$$

holds. It follows from (19) and (20) that the maximal value $\delta_{\mathrm{M}}=\max _{1 \leq \theta \leq \pi} \delta$ of error $\delta$ with respect to $\theta$ depends only on a single number - the ratio $\sigma_{1} / \sigma_{2}$ between the principal summands. Figure 9 illustrates the distribution of this error when doing experiments with a square-shaped sample $\left(a=b=1, a^{\prime}=0.5\right)$, at different values of contact lengths $h$ (in the parentheses we see percentage coverage of the sample contour by contacts $\kappa=\frac{9 h}{2(a+b)} 100 \%$, see Fig. 7).

Thus, the error $\delta_{\mathrm{M}}$ acquires the minimal value under isotropic conductivity (if $\sigma_{1}=\sigma_{2}$, then $\delta_{\mathrm{M}} \leq 0.1 \%$ ), and it increases with an increase in contact lengths as well as recession of the ratio $\sigma_{1} / \sigma_{2}$ from 1 . For instance, if $h=0.05$ (the 


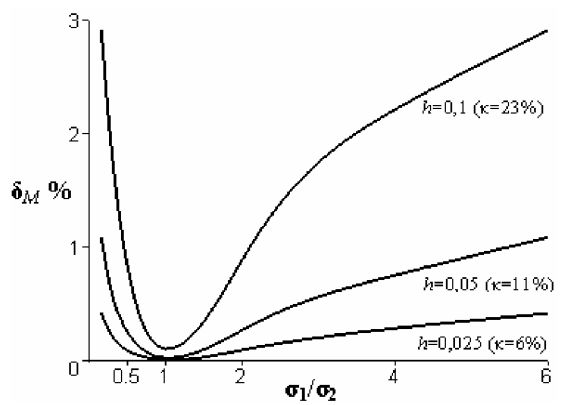

Fig. 9. The dependences of error distributions on the length of contacts $h$ and on the ratio $\sigma_{1} / \sigma_{2}$.

contacts coverage $\kappa=11.25 \%$ of the sample contour) and $1 / 6 \leq \sigma_{1} / \sigma_{2} \leq 6$, then the error does not exceed $1.1 \%$.

\section{Conclusions}

1. The new method for calculating the specific electrical conductivity tensor $\sigma$ of a flat anisotropically conductive medium has been developed, based on the fact that:

a) a linear plane transformation that canonizes an elliptic-type equation changes the physical properties of the sample: the previous anisotropic conductivity becomes isotropic, the specific conductivity of which is coincident with $\sqrt{\operatorname{det} \sigma}$;

b) if the lengths of sample contacts are short as compared with the sample perimeter, then, by alternating the geometrical shape of the sample as well as location of contacts, the ratios between potential differences and current intensities are retained rather stable (Van der Pauw theorem [5]).

2. By applying the Schwartz-Christoffel transformation, we have proved that, in the case of anisotropic conductivity, using a rectangular-shaped sample with five contacts and having done three experiments, it is possible to uniquely find the summands of the specific conductivity tensor. To solve this problem, a system of equations has been constructed and the way of its numerical solution indicated.

3. The volume of calculation is not so large: it takes up about 1 second, employing the usual computational technique.

4. The problem of imitating all the experiments done has been solved. On its basis, the errors of the method have been computed and graphs of their distribution presented.

5. We have established that the errors essentially depend on the relative contact lengths and the degree of anisotropy of the considered substance, i.e., on the ratio $\sigma_{1} / \sigma_{2}$ of the principal summands of tensor $\sigma$. We have defined that in case the sum of all the contact lengths makes up to $23 \%$ of the sample perimeter and $1 / 6 \leq \sigma_{1} / \sigma_{2} \leq 6$, the relative tensor error $\delta_{\mathrm{M}}$ does not exceed $3 \%$, and if $1 / 2 \leq \sigma_{1} / \sigma_{2} \leq 2-\delta_{\mathrm{M}} \leq 1 \%$ and if the medium is isotropic, then $\delta_{\mathrm{M}} \leq 0.1 \%$. 


\section{References}

[1] I. Brila, WSEAS Trans. Commun. 4, 306 (2005).

[2] Ch. Bergemann, J. Appl. Phys. 98, 043707 (2005).

[3] G. Rietveld, C.V. Koijmans, L.C.A. Henderson, M.J. Hall, S. Hannon, P. Warnecke, B. Schumacher, IEEE Trans. Instrum. Meas. 52, 449 (2003).

[4] Y. Sato, S. Sato, Jpn. Appl. Phys. 40, 4256 (2001).

[5] L.J. Van der Pauw, Philips Res. Repts. 13, 1 (1958).

[6] W.L.V. Price, J. Phys. D, Appl. Phys. 5, 1127 (1972).

[7] V. Kleiza, J. Kleiza, Comput. Maths Math. Phys. 32, 1399 (1993).

[8] V. Kleiza, J. Kleiza, Dokl. Akad. Nauk SSSR [Sov. Phys.-Dokl.] 325, 711 (1992). 